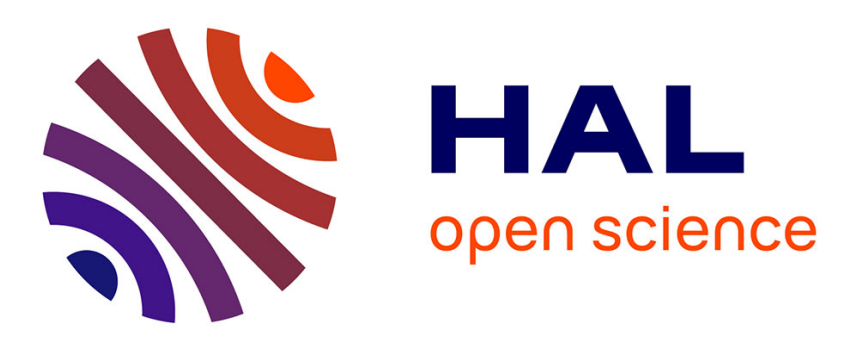

\title{
VORKOMMEN UND HALTUNG DER ÖSTLICHEN HONIGBIENE (APIS CERANA FABR.) IN AFGHANISTAN
}

Peter Schneider, Ahmad Schah Djalal

\section{- To cite this version:}

Peter Schneider, Ahmad Schah Djalal. VORKOMMEN UND HALTUNG DER ÖSTLICHEN HONIGBIENE (APIS CERANA FABR.) IN AFGHANISTAN. Apidologie, 1970, 1 (3), pp.329-341. hal-00890293

\section{HAL Id: hal-00890293 \\ https://hal.science/hal-00890293}

Submitted on 1 Jan 1970

HAL is a multi-disciplinary open access archive for the deposit and dissemination of scientific research documents, whether they are published or not. The documents may come from teaching and research institutions in France or abroad, or from public or private research centers.
L'archive ouverte pluridisciplinaire HAL, est destinée au dépôt et à la diffusion de documents scientifiques de niveau recherche, publiés ou non, émanant des établissements d'enseignement et de recherche français ou étrangers, des laboratoires publics ou privés. 
Apidologie, 1970, 1 (3), 329-341

\title{
VORKOMMEN UND HALTUNG DER ÖSTLICHEN HONIGBIENE (APIS CERANA FABR.) IN AFGHANISTAN
}

\author{
Répartition et élevage de l'abeille orientale (Apis cerana Fabr.) \\ en Afghanistan \\ Peter SCHNEIDER und Ahmad Schah DJALAL \\ Zoologisch-Parasitologisches Institut der Universität, Kabul (Afghanistan)
}

\section{SUMMARY}

DISTRIBUTION AND BREEDING OF THE ORIENTAL BEE (APIS CERANA)

IN AFGHANISTAN

The south-western boundary of the region where Apiscerana is found may take its course through Afghanistan. Here $A$. cerana is mainly living in the south-eastern parts of the country. In the arid areas of the south-west there are no bees living. In the province of Paktia we find wild bees. On the whole route from Gardez to Jamkani as well as in the woodlands of Khost, there are bee colonies kept by indigenous beekeepers. In eastern Afghanistan $A$. cerana is found as far as in the districts of Jalalabad and Nuristan, in the south of Hindu Kush. In the region of Kunar and Nuristan colonies of wild bees mostly are found in crevices and in hollow trees. In Paktia the nests foremost are built in crevices. The most northern and completely isolated area is situated in CentralAfghanistan. There are various beekeeping methods in Afghanistan, but real beekeeping is found in Nuristan and Paktia, only. Here wooden frames are inserted into the mud-walls of the houses from where the hive entrances conduct into the open air. The most important enemies of A.cerana in Afghanistan are hornets and bee-eaters (Merops).

\section{ZUSAMMENFASSUNG}

Die nordwestliche Verbreitungsgrenze von Apis cerena dürfte durch Afghanistan gehen. Hier ist $A$. cerena weitgehend auf die südöstlichen Gebiete beschränkt, während die südwestlichen ariden Gebiete nicht von Bienen besiedelt sind.

In der Provinz Paktia finden sich Wildbienen, und auf der ganzen Strecke von Gardez bis Jamkani sowie in den Waldgebieten von Khost werden Bienen von einheimischen Imkern gehalten, 
Die östlichste Verbreitung von $A$. cerana in Afghanistan liegt südlich vom Hindukusch in den Gebieten von Jalalabad und Nuristan. Lie Wildnester im Kunargebiet und Nuristan sind meist in Felsritzen, vielfach aber auch in hohlen Baumstämmen. In Paktia nisten die Bienen überwiegend in Felsspalten.

Das nördlichste und völlig isolierte Gebiet findet sich in Zentralafghanistan.

Die Haltungsmethoden sind in Afghanistan verschieden, richtige Bienenhaltung wird in Nuristan und Paktia betrieben. In diesen Gebieten werden Holzrè hmen in die Lehmwände der Häuser eingelassen, und die Fluglöcher führen durch diese nach aussen.

Die wichtigsten Feinde der Bienen sind in Afgh anistan Hornissen und Bienenfresser (Merops).

\section{EINLEITUNG}

Apis cerana FABr. (= syn. A. indica FABr.) ist im südlichen Asien weit verbreitet (vgl. Gozтze, 1964). Hiernach dürfte die nordwestliche Verbreitungsgrenze durch Afghanistan gehen. Tats:ichlich mischen sich in Afghanistan orientalische und palaearktische Faunenelemente. Das Vorkommen von A.cerana läuft fast mit der tiergeographischen Grenze zwischen diesen beiden Zonen parallel, wenn man von dem Vorkommen in Zentralafghanistan absieht. In vielen Ländern der Verbreitungszone Asiens wird A. cerana zur Honiggewinnung gehalten, doch sind die Zuchterfolge, die von verschiedenen Autoren geschildert werden, von unterschiedlichem Erfolg. Nach H. Oschmann (1961), sind diese Bienen bei den chinesischen Imkern sehr beliebt, da sie eine grosse Temperaturtoleranz in der Sammelaktivität haben. In Japan wurde A. cerana mit Erfolg in Zucht genommen (I. Okada, T. Sakati und H. Obata (1958). Dagegen berichtet Roepke (1930) von gelungenen und misslungenen Haltungsversuchen auf Java. In Pakistan scheint die Zucht von A. cerana gute Fortschritte zu machen. Das Bee Research Institute of Peshawar hält die einheimischen Bienen in Holzkästen, die nach dem europäischen Muster gebaut sind (mit Mobilbau) [vgl. LATIF et al., 1960]. Um ein Wanderschwärmen der Völker zu vermeiden, wechseln sie den Standort je nach Temperatur und Trachtmöglichkeit. Im Frühjahr 1969 zählten wir an zwei Stellen etwa $15 \mathrm{~km}$ hinter Peshawar jeweils über 80 Bienenvölker. A. cerana wird auch in Kaschmir mit Erfolg zur Honiggewinnung gezüchtet.

Misserfolge in der Haltung sind vor allem in Afghanistan aufgetreten. Die vor einigen Jahren angesetzten Bienenfarmen mit einheimischen Bienen sind aufgelöst worden und an deren Stelle die italienische Rasse von A. mellifica im Süden und die kaukasische Rasse im Norden eingeführt worden; auf diese Versuche wird später noch hingewiesen. In der vorliegenden Arbeit soll nun versucht werden, neben der Verbreitung auch einige Hinweise auf die Haltungsmethoden der einheimischen Imker zu geben. Dies ist deshalb interessant, weil die Bienen in dem hochgebirgigen Afghanistan immer wieder in isolierten Arealen auftauchen und sich so typische Haltungsmethoden entwickelt haben.

Die Untersuchungen wurden 1968-1969 im Rahmen der Partnerschaft der Universitäten Bonn-Kabul durchgeführt. Allen afghanischen Regierungsstellen sei für die Unterstützung in den Grenzgebieten gedankt.

Die Arbeiten wurden angeregt durch Herrn Professor KLofr, Bonn und stehen im Zusammenhang mit Untersuchungen, welche von ihm zusammen mit den Herren Proff. Heran/Würzburg, MARKL/Darmstadt und RUTTNER/Frankfurt-Oberursel mit Unterstützung der Deutschen Forschungsgemeinschaft durchgeführt werden. Unserem Kollegen Dr. H. KLocKenhoff z.Zt. Kabul, sind wir für seine Mitarbeit zu Dank verpflichtet. 


\section{VERBREITUNG}

A. cerana ist in Afghanistan weitgehend auf die süd ̋̈stlichen Gebiete beschränkt, während die südwestlichen ariden Gebiete nicht von Bienen besiedelt sind. A. cerana kommt in Afghanistan in zwei Rassen vor. 1954 aus Kabul von uns (A. S. DJALAL) nach Deutschland verbrachtes $A$. cerana-Material wurde nämlich durch Vermittlung von Professor Lindauer in der Bayerischen Staatssammlung als $A$. peroni und $A$. picea determiniert *

In Abb. 1 sind die Funde in schräger Schraffur eingetragen. Selbstverständlich konnten nicht alle Gebiete von uns selbst untersucht werden, da durch die unzureichenden Strassenverhältnisse viele Bienenvorkommen unzugänglich sind. So mussten wir uns oft auf die Aussagen der Bevölkerung verlassen. In der Provinz Paktia fanden wir Wildbienen und auch von einheimischen Imkern gehaltene Bienen auf der ganzen Strecke von Gardez bis Jamkani. Meist befindet sich nur ein Imker in einem Dorf. Ebenso fanden wir von Jamkani bis in die Waldgebiete von Khost (Matoon) immer wieder sichere Nachweise. Die östlichste Verbreitung in Afghanistan liegt in den südlichen Ausläufern des Hindukusch in den Vorgebirgen und Gebirgen von Nuristan. So fanden wir Bienen ab Jalalabad über den Dario Nur, das Dara-e-Mazar (bei Nurgal), Shaga sarai, Pech-Tal, Schitral, Barikot und Kamdesch. Wildnester, die wir in Jalalabad sahen, befanden sich in Kellerlöchern und in dem Dachstuhl eines Hauses. Die Wildbienennester im Kunargebiet und Nuristan sind zum überwiegenden Teil in Felsritzen (vgl. KLofT und SCHNEIdER 1969), vielfach aber auch in Maulbeerbäumen und anderen hohlen Stämmen. In Paktia nisten die Bienen meist in Felsspalten. Die nördlichste Verbreitungsgrenze des Paktiagebietes ist Charkh im Logar-Tal. Hier nisten die Bienen ausschliesslich in Felsspalten. Dieses Gebiet ist waldfrei. An den Hängen, wo Wildbienen vorkommen, stehen vereinzelt wilde Mandelb̈̈ume (Amyctalis spec), die im Frühjahr die Haupttracht darstellen, während im Sommer Blattlaushonig, vor allem von den Maisfeldern eingetragen wird. In Kabul selbst sollen nach Berichten des Landwirtschaftsministeriums auch schon einheimische Bienen gesehen worden sein, doch halten wir dies für verwilderte Bienen aus Zuchtversuchen.

Das nördlichste und auch völlig isolierte Gebiet fanden wir in Centralafghanistan. Es waren dies die Gebiete um Deh Kundi und die Waldgebiete von Sharistan. Es wurde uns zwar wiederholt berichtet, dass auch in der Provinz Ghor (westlich von Chakhcharan) Bienen vorhanden seien, doch konnten wir es nicht mit Bestimmtheit erfahren. Das gleiche gilt auch für die Vorkommen in den Gebirgen des oberen Laghman. Letzteres scheint aber wahrscheinlich, da es klimatisch etwas den Nuristanbergen gleicht.

Bei allen einheimischen Imkern, die wir auf unseren Exkursionen besuchten, fragten wir immer wieder nach $A$. dorsata, der Riesenbiene und nach Apis florea. der Zwergbiene. Während erstere immer wieder verneint wurde und auch diu Waben nicht bekannt zu sein schienen ( in Peshawar, Pakistan, konnten wi Apis dorsata beobachten), wurde das Vorkommen von A. florea immer wieder

* Nach der heute als unhaltbar geltenden Terminologie von maA (1953). Die beiden genannten Formen sind als Subspecies von $A$. cerana aufzufassen. 


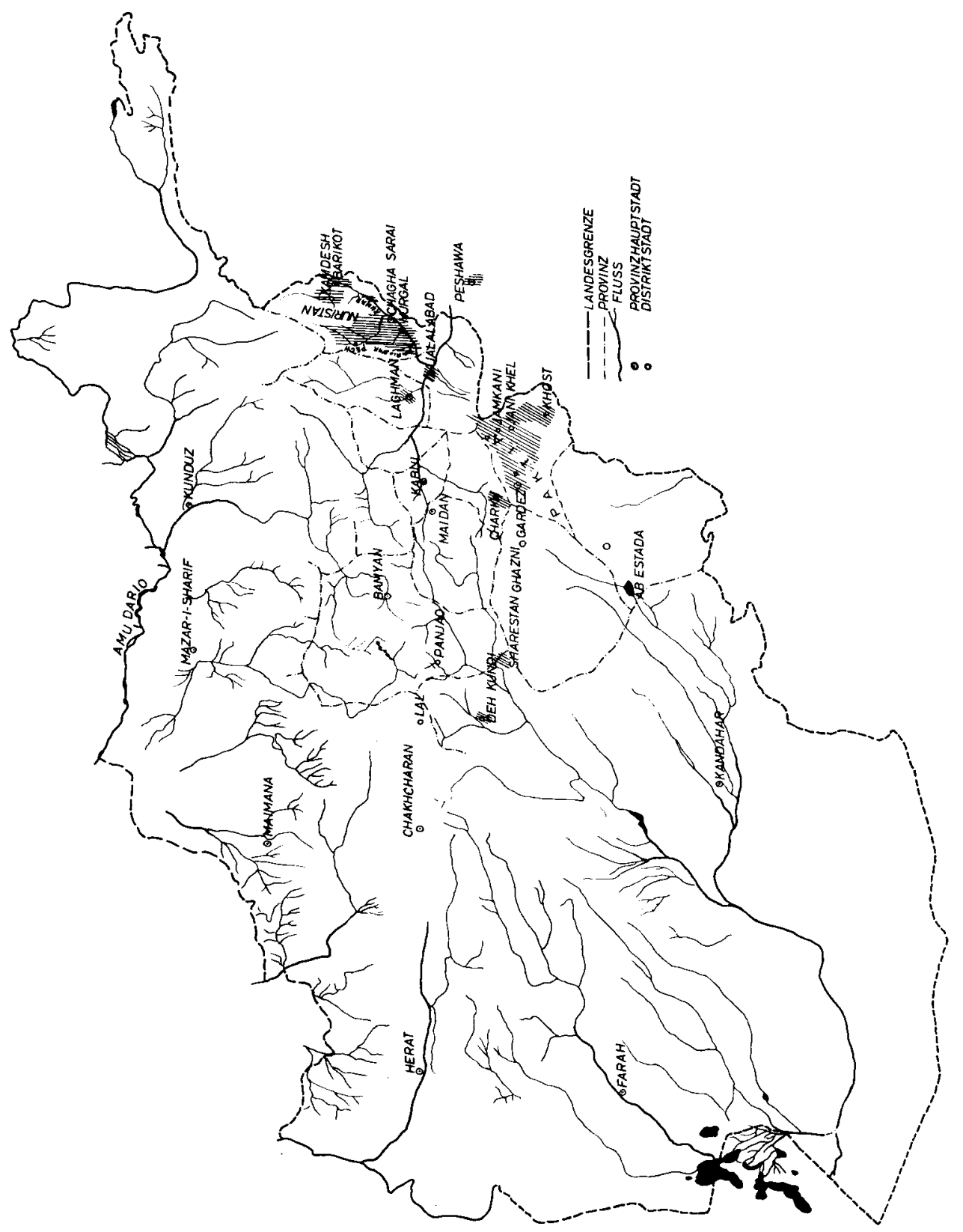

Aвв. 1. - Verbreitungskarte von Apis cerana in Afghanistan

Fig. 1. - Carte de la rëpartition d'Apis cerana en Afghanistan

Lexique : Landesgrenze $=$ trontières. Provinz : province. Fluss $=$ rivière. Provinzhaupstadt $=$ capitale de province. Distriktstadt $=$ lieu de district. 
bestätigt. Eine sichere Auskunft erhielten wir von dem Leiter der Bienenfarm in Khost, der selbst 2 Jahre diese Bienen gehalten hatte. Er beschrieb sowohl die Bienen als auch den Wabenbau genau. Selbst haben wir aber Apis florea noch nicht gesehen.

\section{HALTUNG UND ZUCHT}

Die oben beschriebenen Bienengebiete sind nicht nur lokal oft stark getrennt, sondern werden auch von verschiedener afgh. Bevölkerung bewohnt. (Paktia, Pachtunen; Nuristan, Nuristani; Centralafghanistan, Hazarah). So fanden wir verschiedene Methoden der Ausnutzung und Haltung von Apis cerana. Im Pech-Tal fanden wir mehrfach Wildbienen ab Sinhahi, dortselbst in einem Maulbeerbaum, aber nirgends Anzeichen einer Imkerei. Der Honig ist zwar bekannt und man fährt mit einem Stock in die Waben, um so etwas Honig zu gewinnen. Wird ein Baum gefällt, dann lässt man den Honig aus den Waben laufen und benutzt ihn zum Süssen des Tees. Richtige Bienenhaltung wird erst in Nuristan betrieben.

Im oberen Pech-Tal (Warma) mit seiner nuristanischen Bevölkerung fanden wir 2 Imker mit je zwei Völkern. Der eine Imker hielt die Bienen in einem ausgehöhlten Baumstumpf, wie er als Trommel benutzt wird. Der andere Imker hatte die Bienen in einem Tontopf untergebracht. Abb. 2 zeigt diesen Tontopf

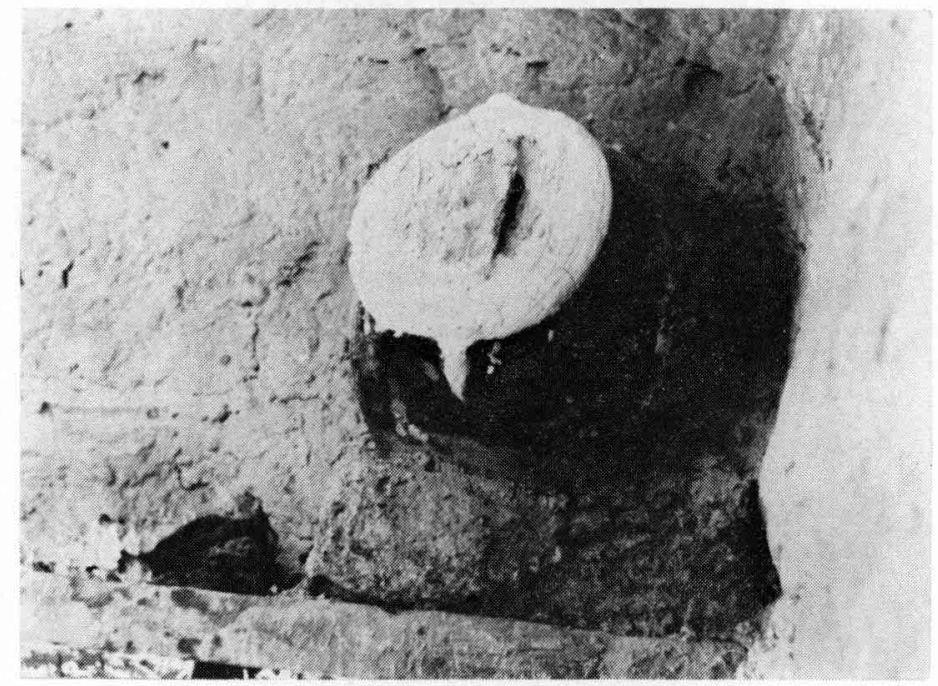

Aвв. 2. - Innenraum eines nuristanischen Wohnraumes mit einem in die Wand eingelassenen Tonkrug Der Krug ist mit einem Deckel verschlossen.

FIG. 2. - Intérieur d'une habitation nuristanaise avec un pot d'argile encastré dans le mur. Le pot est muni d'un couvercle.

im Innern des Hauses. Der Krug ist mit der Bodenöffnung in die Aussenmauer eingelassen und mit Lehm bis auf ein kleines Flugloch zugeschmiert. Der sich etwas verjüngende Krug ist mit einem Deckel verschlossen, der einen schmalen Spalt frei lässt. 
Ergebnisse einer früheren Exkursion (mündliche Mitteilung von G. KüHNERT) zeigten, dass in einem Ort nahe bei Kamdesch Bienen ebenfalls in Häusern gehalten werden. Die Nester befinden sich in Holtztruhen, die im Innern der terassenförmig übereinander gebauten Häuser zu ebener Erde stehen und deren Flugloch nach aussen durch die Wand geht. Ähnliche Haltungsmethoden fanden wir immer wieder in der Provinz Paktia. Hier werden Holzrahmen in die Lehmwände der Häuser eingelassen. Aussen wird der Bienenkasten mit Lehm verputzt, durch die die 1-2 Fluglöcher (5-7 cm $\varnothing)$ führen. Die Innenseite des Kastens zum Wohnraum oder Stall ist mit einem Brett verschlossen, das ebenfalls mit Lehm verschmiert ist. Die Grösse der Rahmen schwankt zwischen $80-100 \mathrm{~cm}$ Länge und 20-45 cm Höhe. Halterungen für die Waben gibt es nicht; diese werden von den Bienen frei gebaut. Auf Abb. 3 kann man die Fluglöcher einheimischer Bienen

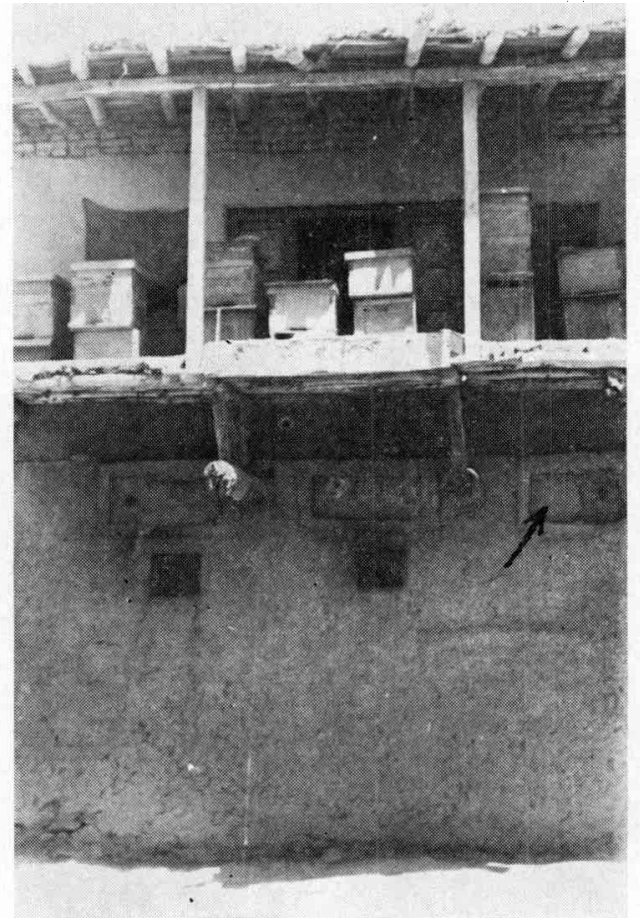

Aвв. 3. - Haus eines Imkers in Gardez

Unter dem Balkon sieht man die Einfluglöcher der Apis cerana mit ihren Holzrahmen, auf dem Balkon Kästen mit der europäischen Honigbiene.

Fig. 3. - Maison d'apiculteur à Gardez

Sous le balcon se trouvent les trous de vol d'Apis cerana avec les cadres de bois, sur le balcon les ruches d'abeilles européennes.

sehen. Die Aufnahme wurde in Gardez bei einem Imker gemacht, der sowohl einheimische als auch "Italiener " hielt. Abb. 4 zeigt die Anlage der Bienennester 

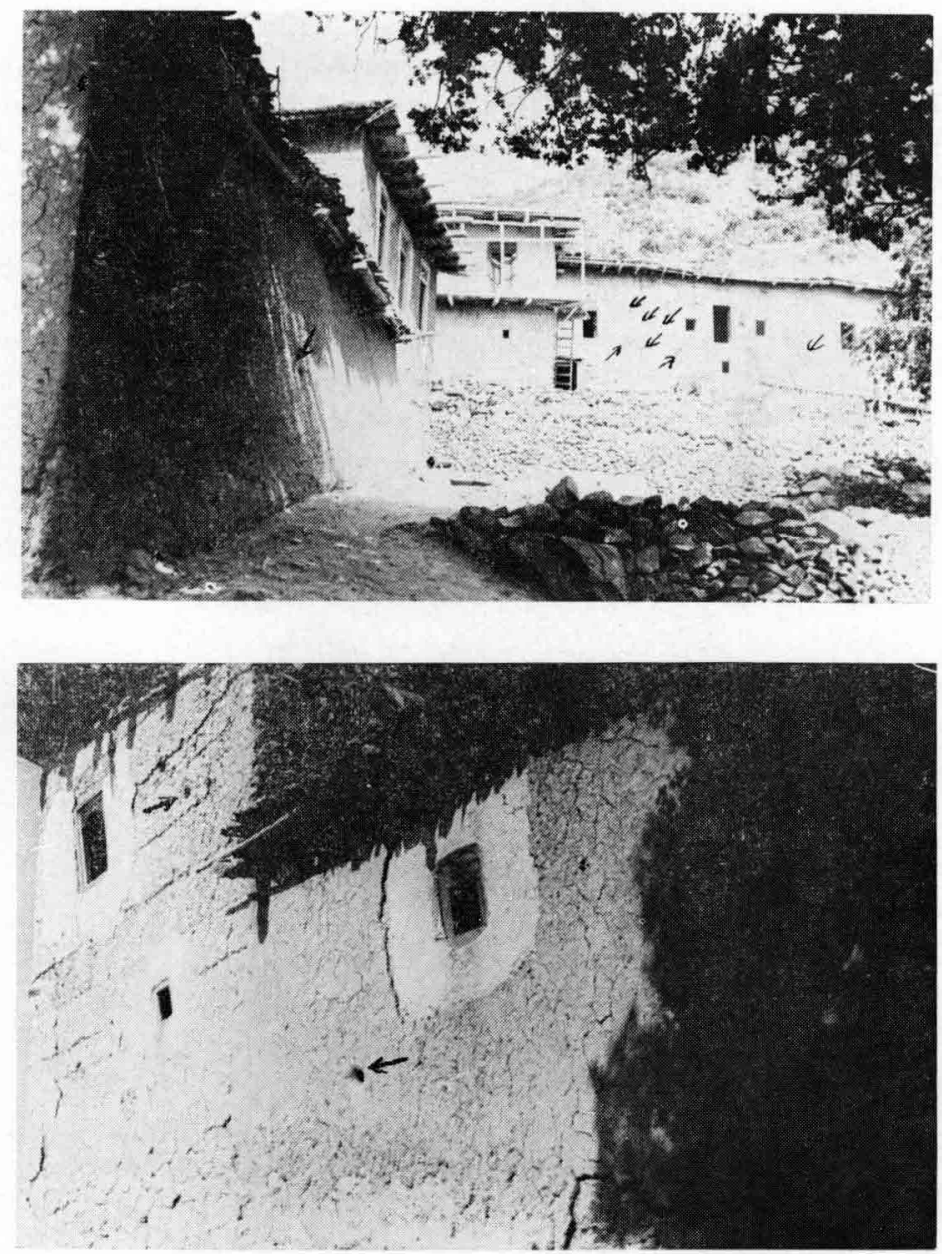

Aв8. 4. - Das Gehöft des Herrn Alef-u-Din in Garamay

Die Pfeile zeigen die Einfluglöcher in der Hauswand an.

Fig. 4. - Ferme de M. Alef-u-Din à Garamay

Les flèches indiquent les trous de vol dans le mur.

des vielleicht grössten afghanischen Imkers, der über 60 Völker hatte. Speziell dieser Imker, Herr Alef-u-Din aus Garamay (15 km östlich von Jamkani), gab uns wichtige Informationen aus seiner über 40-jährigen Praxis. Das Gehöft Garamay liegt auf einer Höhe von $1960 \mathrm{~m}$. Seine Bienenkästen sind in der oben beschriebenen Weise in der Hauswand in verschiedenen Höhen eingelassen. Die Länge dieser Kästen beträgt $85 \mathrm{~cm}$ bei einer Höhe von $20-25 \mathrm{~cm}$. Die Tiefe schwankt mit der Dicke der Hauswand. Als Winternahrung lässt Herr Alef-u-Din eine volle Honigwabe in jedem Nest, das Flugloch wird verkleinert und um die Waben Ziegenwolle geschichtet. In sehr kalten Wintern werden kleine Öfen aufgestellt. Während die anderen Imker oft sehr grosse Verluste haben (wohl auch durch zu wenig Futterzugabe im Winter), bekommt dieser Imker die Bienen gut über 
den Winter. Seine Völker schwärmen einmal im Jahr. Die Schwärme fängt er mit einem speziell zu diesem Zweck konstruierten Korb wieder ein, während sonst in Afghanistan nur ein Sack zum Einfangen verwendet wird. Neu eingefangene Völker werden mit einer vollen Honigwabe aus einem anderen Nest versorgt. Die Bienen gewöhnen sich so schnell an das Nest.

Eine völlig andere Methode lernten wir in den Nuristan vorgelagerten Bergen kennen. Dieses Gebiet befindet sich etwa 2 Tagesmärsche von Nurgal das Dara-eMazar aufwärts. Wir erhielten auch unser erstes im Haus gehaltenes Volk aus dieser Gegend, welches zur Weiteruntersuchung nach Oberursel/Taunus an das Institut für Bienenkunde versandt wurde. In dieser Gegend werden die Bienen in Tontöpfen (jetzt in Oberursel/Taunus) gehalten (Abb. 5). Das frisch eingefangene Volk wird

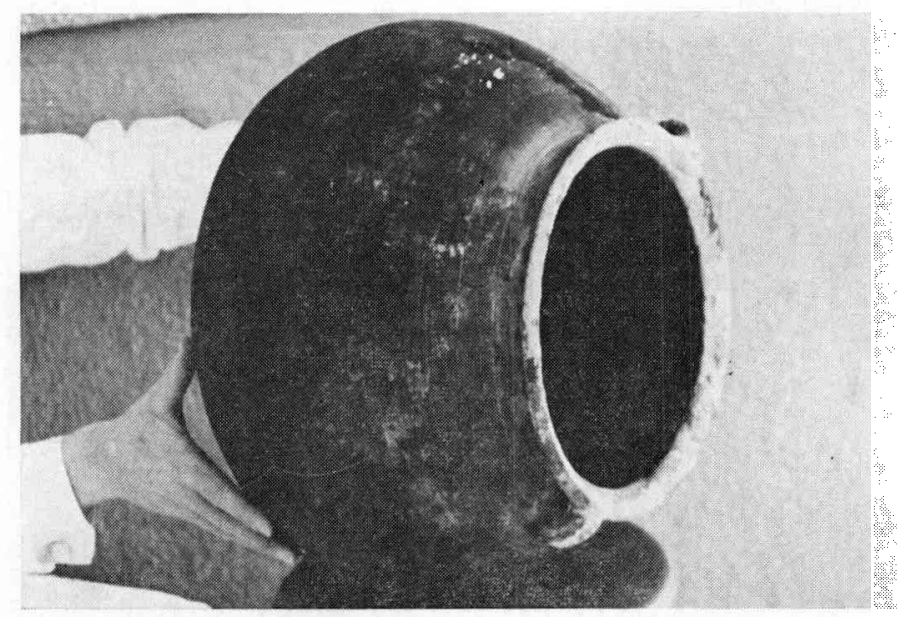

Aвв. 5. - Tontopf aus Ostafghanistan, der als Bienenwohnung verwendet wird

Die Oeffnung wird verschlossen und der Topf in wagrechter Stellung in die Hauswand eingelassen. Inhalt : Etwa 28 Liter.

Fic. 5. - Poterie de l'Afghanistan oriental, utilisêe comme ruche

On ferme l'ouverture et on introduit la marmite en position inclinée dans le mur de la maison. Contenance environ 28 litres.

in diesen Topf, der mit Zucker bestrichen ist, eingesetzt. Vorher wurde ein Loch in den Boden des Topfes geschlagen. Dann wird die Öffnung des Topfes mit einem Tuch verschlossen und dieser in waagerechter Stellung entweder ins Fenster gehängt oder in die Mauer eingelassen. Handelt es sich um ein neues Volk, dann wird vor das Loch im Boden, das jetzt als Flugloch dient, ein Brettchen befestigt, das nur ein Ausflugloch für die Arbeiterinnen hat. Die Königin - Maleka - ist in ihrer Grösse und Bedeutung bekannt. Sehr seltsam erschien uns die Auskunft, dass in diesem Gebiet alle Imker im Winter Rinder- oder Hammelfett als Nahrung für die völlig abgeernteten Völker verwenden. Der Mann, der uns das oben erwähnte Volk besorgte, behauptete, dass er auf diese Wiese schon 4 Jahre ein Volk durch den Winter bekommen hätte.

Der einzige Imker im Logartal (Charkh) hatte eine etwas andere Form für seine Kästen entwickelt. Er hatte sich zum Teil auf dem Dach oder zu ebener Erde 
Holzkästen gebaut, die er mit einer ca. $8 \mathrm{~cm}$ dicken Lehmschicht verkleidet hatte (Abb. 6). Seine Völker schwärmen oft bis 2 mal pro Jahr. Im Winter füttert er seine Bienen mit Zucker.
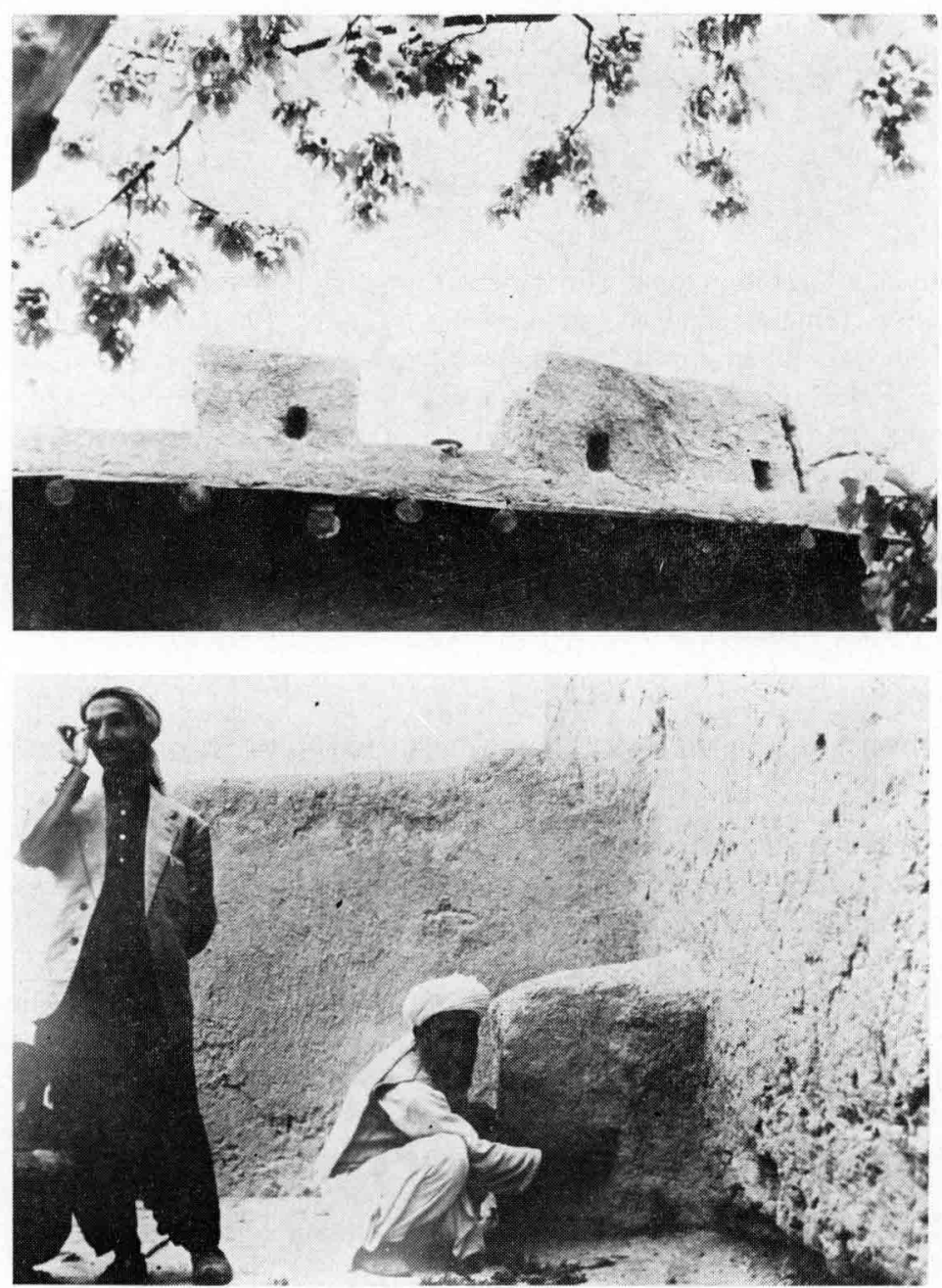

Aв8. 6. - Der einzige Imker im Iogarial

Hält seine Bienen in freistehenden Holzkästen, die mit einer $8 \mathrm{~cm}$ dicken Lehmschicht gegen Kälte und Hitze isoliert sind, zu ebener Erde und auf dem Dach.

Fig. 6. - Seul apiculteur de la vallée du Logar

Il élève ses abeilles dans des ruches individuelles, isolées de la chaleur et du froid par une couche d'argile de $8 \mathrm{~cm}$ d'épaisseur, au rez-de-chaussée et sur le toit.

Eine sehr primitive Haltungsweise wurde uns aus dem nördlichsten Verbreitungsgebiet - in Sharistan — bekannt. Im allgemeinen wird der Honig in diesem Gebiet (auch etwas in Deh Kundi) durch Nutzung der wildlebenden Bienenvölker 
gewonnen. Der so gewonnene Honig gelangt manchmal bis nach der Distriktstadt Panjao.

Mehrfach wurde uns auch mitgeteilt, dass einige Leute Bienen halten.

$\mathrm{Zu}$ diesem Zweck werden Bienenbäume gefällt, der Stamm um das Nest zurechtgestutzt und dieses Baumnest dann am Haus oder im Garten aufgehängt. Im Winter wird es völlig abgeerntet.

\section{FEINDE DER BIENEN}

In einer früheren Arbeit wurde schon auf die Bedeutung der Hornissen als Bienenfeinde hingewiesen (KLOFT und SchneIDER, 1969). Dieser Feind spielt aber erst ab September in Afghanistan eine grössere Rolle, während die Bienenzuchten in Pakistan während der ganzen Saison von Hornissen bedroht sind. Zu diesem Zweck hat man Leute angestellt, die nur anfliegende Hornissen vernichten. Ein sehr starker Feind der Imker, speziell im Logar-Tal, sind die Bienenfresser (Merops). Der Imker aus Charkh sagte uns, dass er manchmal bis zu 10 dieser Vögel pro Tag abschiesst. In manchen Gebieten spielen auch die Ameisen eine Rolle, doch gelingt es den Imkern meistens mit DDT, das sie rund um die Nester streusn, ein Eindringen in den Stock zu verhindern.

\section{VER WENDUNG UND WIRTSCHAFTLICHE BEDEUTUNG DER IMKEREI}

Die ersten staatlichen Bienenzuchtversuche in Afghanistan wurden vor etwa 100 Jahren in Kabul durchgeführt. Als Bienenkästen verwendete man die auf S. 326 erwähnten Tontöpfe. Die Bienen konnten aber weder an der Flucht gehindert werden, noch war der Honigertrag gross. Ähnliche Erfahrungen machte das Landwirtschaftsministerium am Ende der 50 er Jahre. Man legte grosse Bienenfarmen mit $A$. cerana an (bis zu 60 Völkern). Der Ertrag soll pro Volk ein halbes Zer (ca. 7 Pfund) nicht überschritten haben. Aus den Berichten (Abdul Rauf, 1964) geht weiter hervor, dass die Bienen ihre Kästen immer wieder verliessen. Man hat dann die Zucht einheimischer Bienen aufgegeben und die italienische Rasse der europäischen Honigbiene eingeführt. Das Misslingen der $A$. cerana-Zucht liegt nach unserer Meinung an der zu grossen Zahl angesetzter Völker in Gebieten, die nicht genügend Tracht boten. Nach Lindauer (1956) fliegt die europäische Honigbiene gut bis $6 \mathrm{~km}$, zeigt aber auch bei Entfernungen bis zu $11 \mathrm{~km}$ noch richtungsweisende Tänze. Lindauer beobachtete auf Ceylon bei $A$. cerana richtungsweisende Tänze nur bis maximal $750 \mathrm{~m}$. Tatsächlich waren die Bienenzuchtversuche in unbewaldeten Gebieten gemacht worden. Die Leute des Bee Research Instituts von Peshawar teilten uns mit, dass sie die gleichen Schwierigkeiten gehabt hätten, bis sie dazu übergegangen seien, die Standorte mit der Jahreszeit zu ändern. Nun bleiben die Völker in den Stöcken und die Ausbeute ist befriedigend.

Der Honig wird von den Afghanen gleich welches Stammes oder welcher Rasse nur zum Süssen des Tees verwendet. Nirgends erhielten wir einen Hinweis auf Verwendung als Brotaufstrich oder als Backzutaten. Allerdings benutzt man den Honig als Medizin gegen Infektionskrankheiten, Magenbeschwerden und Fie- 
ber. Sieht man von den Bienenfarmen des Landwirtschaftsministeriums mit seinen europäischen Bienen ab, dann kann man sagen, dass die Imkerei keinerlei wirtschaftliche Bedeutung für das Land hat. Abgesehen von der Zucht des Herrn Alef-u-Din in Garamay, haben die einheimischen Imker selten mehr als 4-6 Völker. Manche einheimischen Imker halten neben den " wilden " Bienen auch importierte (vgl. Abb. 3). Dies beobachteten wir mehrfach in Gardez. Leider ist man aber nicht dazu übergegangen, die verschiedenen Zuchtmethoden zu kombinieren. So hält z.B. ein Imker in Gardez die italienische Rasse von $A$. mellifica in nachgebauten europäischen Bienenkästen mit Waben, während er gleichzeitig seine einheimischen Bienen, die er sogar höher einschätzt als die "Ausländer ", weil sie zwar wenig, aber besseren Honig machen, in der vorher beschriebenen Weise - Kästen in der Hauswand - hält. Apis cerana in Wabenrahmen bauen zu lassen oder in richtigen Bienenkästen, wie sie für die "Italiener" benuzt werden, wurde noch nicht versucht. Zwei weitere Imker aus diesem Ort handelten ähnlich.

Der Honig in den Naturwaben der Cerana-Völker wird nicht durch Schleudern gewonnen. Man legt die volle Honigwabe auf einen Teller und lässt den Honig auslaufen. Manchmal wird die Wabe auch geknetet und der Honig in Ofennähe gewonnen.

Die Ausbeute eines genutzten $A$. cerana-Volkes beträgt in der Regel 4-6 Zer $(28-42 \mathrm{~kg})$ pro Janr. Die auf den Farmen des Landwirtschaftsministeriums gewonnene Honigmenge wird auf 7-8 Zer $(42-49 \mathrm{~kg})$ pro Jahr geschätzt.

Wird die Honiggewinnung von den einheimischen Imkern auch kommerziell ausgenutzt ( $450 \mathrm{~g}$ kosten 50-60 Afs, ca. 2,50-3,00 DM), so gewannen wir doch den Eindruck, dass die Bienen nie aus rein kommerziellen Gründen gehalten werden, denn auf unseren Exkursionen stellten wir immer wieder fest, dass alle Imker grosse Zuneigung zu ihren Bienen empfinden. So wird es auch verständlich, dass es für uns sehr schwer, ja meist fast unmöglich war, Völker von einheimischen Imkern zu bekommen. Alle Völker, die wir bekamen und nach Deutschland schickten, waren Wildfänge, für die die Imker keine Verwendung hatten. Ein Bienenstock ist für afghanische Verhältnisse sehr teuer, nämlich 1000 bis 1700 Afs., das wären etwa 50, - bis 85, - DM. Wenn auch die europäische Honigbiene in den staatlichen Farmen die einheimische Biene verdrängt hat, wird es sicher noch sehr lange dauern, bis sie auch in den abgelegenen Dörfern gehalten wird oder gar die einheimische aus der Haltung verdrängt.

Eingegangen im Februar 1970. Reçu pour publication en février 1970.

\section{RESUMÉ}

Le présent travail cherche à donner, en plus de la répartition d'Apis cerana, quelques indications sur les méthodes d'élevage de l'apiculture indigène.

La limite nord-ouest d' $A$. cerana, très répandue en Asie du sud, devrait passer par l'Afghanistan. Ici $A$. cerana est largement répandue dans les limites des régions sud-est, tandis que les régions sud-ouest arides en sont dépourvues. 
Dans la province de Paktia, on trouve des abeilles sauvages et dans tout l'espace compris entre Gardez et Jamkani, elles sont élevées par les apiculteurs indigènes. On en trouve également des signes certains entre Jamkani et la région forestière de Khost.

La répartition orientale d'A. cerana en Afghanistan s'étend aux contreforts méridionaux de l'Hindukusch, dans les promontoires et montagnes du Nuristan. On a trouvé ainsi des abeilles à partir de Jalalabad, dans les régions suivantes : Dara-e-Nur, Dara-e-Mazar, Shagasarai, Pechtal, Schitral, Barikot et Kamdesch.

Les nids sauvages, dans les régions de Kunar et du Nuristan, se trouvent pour la plupart dans les fissures de rochers, souvent aussi dans des troncs d'arbre creux. Dans le Paktia, les abeilles nidifient surtout dans les fentes de rochers. La limite septentrionale de répartition dans la région de Paktia est Charkh, dans la vallée du Logar. Dans cette région dépourvue de forêts, les abeilles nidifient également dans les fentes de rochers.

La zone la plus septentrionale, complètement isolée, se trouve dans l'Afghanistan central. Ce sont les régions boisées de Sharistan et les régions autour de Deh Kundi.

Les méthodes d'élevage diffèrent suivant les régions mentionnées. Bien que le miel soit connu dans la vallée du Pech, on n'y rencontre aucune trace d'apiculture. Le véritable élevage d'abeilles n'est pratiqué dans ces régions que dans le Nuristan. Dans la haute vallée du Pech, les abeilles sont élevées, la plupart du temps, dans des récipients d'argile à l'intérieur des maisons, le vol vers l'extérieur se faisant à travers le mur. Dans le Kamdesch, les abeilles sont élevées dans des cadres de bois, également à l'intérieur des maisons. Les trous de vol passent à travers le mur. Des méthodes d'élevage similaires ont cours dans la province de Paktia. Les cadres de bois sont encastrés dans les murs de torchis où se trouvent les trous de vol vers l'extérieur. La partie intérieure de la caisse servant de logement ou d'abri est fermée à l'aide d'une planche. Il n'y a pas de support pour les rayons, ceux-ci sont construits librement par les abeilles.

Avec l'aide du Ministère de l'Agriculture d'Afghanistan, on a tenté d'installer Apis cerana dans de grosses exploitations équipées de ruches à hausses multiples. Mais les abeilles désertaient continuellement leurs ruches. On a donc abandonné l'élevage des abeilles indigènes dans ces exploitations et importé des abeilles italiennes et caucasiennes. L'échec de l'élevage est peut-être dû au fait que des colonies ont été placées en grand nombre dans des régions qui n'offraient pas des ressources mellifères suffisantes.

Les principaux ennemis des abeilles en Afghanistan sont les frelons et les " guêpiers "(Merops).

Entre autres usages, le miel est souvent utilisé, en Afghanistan, comme remède contre diverses maladies.

\section{LITERATUR}

Goetze G. K. L., 1964. Die Honigbiene in natürlicher und künstlicher Zuchtauslese. Beih. Z. Angew. Entomol., (19), (20).

Kloft W., Schneider P., 1969. Gruppenverteidigungsverhalten bei wildlebenden Bienen. Naturwiss., 56, 219.

Latif A., Qayyum A., Manzoor ul Hag, 1960. Multiple and twoqueen Systems in Apis indica. Bee World, 41, 201-210.

Lindauer M., 1956. Uber die Verständigung bei indischen Bienen. - Z Zergl. Physiol., 38, 521-557.

MAA T., 1953. An Inquiry into the systematics of the tribus Apidini or Honeybees (Hym.). Treubia, $21,525-640$. 
Oxada I., T. Sakati und Obata H., 1958. On the habits of japanese honeybee.-XXVII. Intern. Beekeeping Congr. Bologna-Rom, 5, 23.

Oschmann H., 1961. Bienenzucht und Bienenhaltung in China. - Arch. Bienenkunde, 38, 27-32. Raur Abdul, 1964. Die Honigbiene (persisch). - Landwirtschaftsministerium Kabul.

ROEPKE W., 1930. Beobachtungen an indischen Honigbienen. - Meded. Landbouwhoogesch.; Wageningen, 34, 1-22.

Swanston D., 1969. Afghanistan Beekeeping. - Amer. Bee J., 20-21.

Anschrift der Verfasser :

Dr. P. Schneider, Institut für Angewandte Zoologie 53 BONN An der Immenburg 1

Dr. A.S. Djalal,

Zoologisch-Parasitologisches Institut

KABUL P.U.B. 21 